\title{
Wide-Awake Approach for Flexor Digitorum Superficialis Tendon Transfer Followed by Early Active Mobilization
}

\author{
Takuma Kuroda ${ }^{1,2}$ Koji Moriya ${ }^{1}$ Naoto Tsubokawa ${ }^{1}$ Hiroko Narisawa ${ }^{1}$ Yutaka Maki \\ Katsunori Inagaki ${ }^{2}$ Takae Yoshizu ${ }^{1}$ \\ ${ }^{1}$ Niigata Hand Surgery Foundation, Niigata, Japan \\ 2Department of Orthopedic Surgery, Showa University School of \\ Medicine, Tokyo, Japan

\begin{abstract}
Address for correspondence Takuma Kuroda, MD, PhD, Department of Orthopedic Surgery, Showa University School of Medicine, 1-5-8 Hatanodai, Shinagawa-ku, Tokyo, 142-8666, Japan (e-mail: tk071210@yahoo.co.jp, tk071210@gmail.com).
\end{abstract}

Indian J Plast Surg 2021;54:338-343.

\begin{abstract}
Keywords

- tendon injuries

- early active mobilization

- flexor digitorum superficialis

- tendon transfer

- wide-awake approach

Background The standard clinical practice to treat closed ruptures of the flexor digitorum profundus (FDP) tendons includes free tendon grafting; however, it is not suitable when the muscle amplitude of the ruptured FDP is not sufficient. We report outcomes of six patients who underwent flexor digitorum superficialis (FDS) tendon transfer of the ring finger using the wide-awake approach to repair the closed rupture of the FDP tendon of the little finger in zone 3 or 4.

Methods The patients were identified by reviewing our institutional billing records from January 2012 to October 2019 for the International Classification of Disease 10 code M66.3 that describes the diagnosis as "spontaneous rupture of flexor tendons." Results The patients comprised two men and four women with an average age of 72.2 years (standard deviation [SD], 8.4 years). All patients were hospitalized after surgery to undergo early active mobilization. The average total active motion at the final evaluation was $201.8^{\circ}$ (range: $85-248^{\circ}$ ). According to Strickland's criteria, outcomes were excellent for two, good for three, and poor for one patient. No patients complained about the ring finger.

Conclusion These results suggest that FDS tendon transfer is recommended when the muscle amplitude of the ruptured FDP is insufficient. We believe that the wide-awake approach and early active mobilization may contribute to satisfactory outcomes.
\end{abstract}

\section{Introduction}

Closed ruptures of the flexor digitorum profundus (FDP) tendons are rare and often require surgical treatment. Free tendon grafting is the standard clinical practice; however, it is not suitable when the muscle amplitude of the ruptured FDP is insufficient. ${ }^{1}$ The flexor digitorum superficialis (FDS) of the other finger is used as an available motor for the ruptured FDP tendon in this situation. FDS tendon transfer is not a new operative procedure. Mayer introduced this technique, which he called "the pedunculated graft."'Although this tendon transfer procedure has been reported to be useful, some surgeons do not prefer this method, because it may be difficult to determine the appropriate tension of the transferred tendon. ${ }^{3-5}$ Recently, the wide-awake approach for hand surgery has become widespread. The simplicity, safety, published online

September 16, 2021
DOI https://doi.org/

10.1055/s-0041-1734577 ISSN 0970-0358

\section{(C) 2021. Association of Plastic Surgeons of India.}

This is an open access article published by Thieme under the terms of the Creative Commons Attribution-NonDerivative-NonCommercial-License, permitting copying and reproduction so long as the original work is given appropriate credit. Contents may not be used for commercial purposes, or adapted, remixed, transformed or built upon. (https://creativecommons.org/licenses/by-nc-nd/4.0/).

Thieme Medical and Scientific Publishers Pvt. Ltd. A-12, 2nd Floor, Sector 2, Noida-201301 UP, India 
and convenience of this technique provides benefits for surgeons and patients. ${ }^{6}$ Of all the operations performed with the wide-awake approach, flexor tendon surgery has been the most improved. The wide-awake approach is useful for flexor tendon surgery, because it allows surgeons to adjust the tension of the transferred tendon during active movement, in order to confirm that the transfer is not overly tight or loose before skin closure. ${ }^{7}$

The purpose of the study was to evaluate the outcomes of the FDS transfer that used the wide-awake approach, followed by early active mobilization, for closed rupture of the FDP tendon.

\section{Materials and Methods}

This study was a retrospective review of a case series. The potential participants were identified by searching our institutional billing records from January 2012 to October 2019 for International Classification of Disease 10 code M66.3 that describes the diagnosis as "spontaneous rupture of flexor tendons." The following inclusion criteria were applied: (1) a closed rupture of the FDP tendon of the little finger, with or without a concomitant FDS tendon rupture in zone 3 or 4; (2) use of the FDS tendon of the ring finger for tendon transfer; (3) operation under local anesthesia or wrist block; and (4) rehabilitation with early active mobilization. Patients with multiple tendon ruptures were excluded. The clinical characteristics and injury details of the study participants were retrieved from the medical and operative records, which included simple sketches of the surgical procedures. All participants provided written informed consent before their participation in the study. This study was approved by the Ethics Committee of the Niigata Hand Surgery Foundation (hospital approval number: R2-1). The study was conducted according to the principles of the World Medical Association Declaration of Helsinki.

\section{Surgical Procedure Selection}

When the proper maximum amplitude of the proximal ruptured FDP tendon stumps was $15 \mathrm{~mm}$ or more, we figured that the ruptured tendon could serve as a motor for the graft, and a free tendon graft using the palmaris longus tendon was performed. ${ }^{1}$ When the proper maximum amplitude of the ruptured FDP tendons stumps was less than $15 \mathrm{~mm}$, either a tendon transfer with the FDS tendon of the ring finger or an end-to-side tendon transfer with the FDP of the ring finger was performed based on the surgeon's preference.

\section{Surgical Technique}

All operations were performed under the wide-awake approach. Each patient received an injection of 10 to $25 \mathrm{~mL}$ in total of $1 \%$ lidocaine with $1: 100,000$ epinephrine delivered to the skin-incision region ( - Fig. 1). Wrist blocks were also used in some patients, based on the surgeon's preference. We usually used a pneumatic tourniquet to control bleeding for the first 20 to 30 minutes of surgery until an adequate epinephrine effect was obtained. A zig-zag skin incision was made around the ruptured site in the palm,

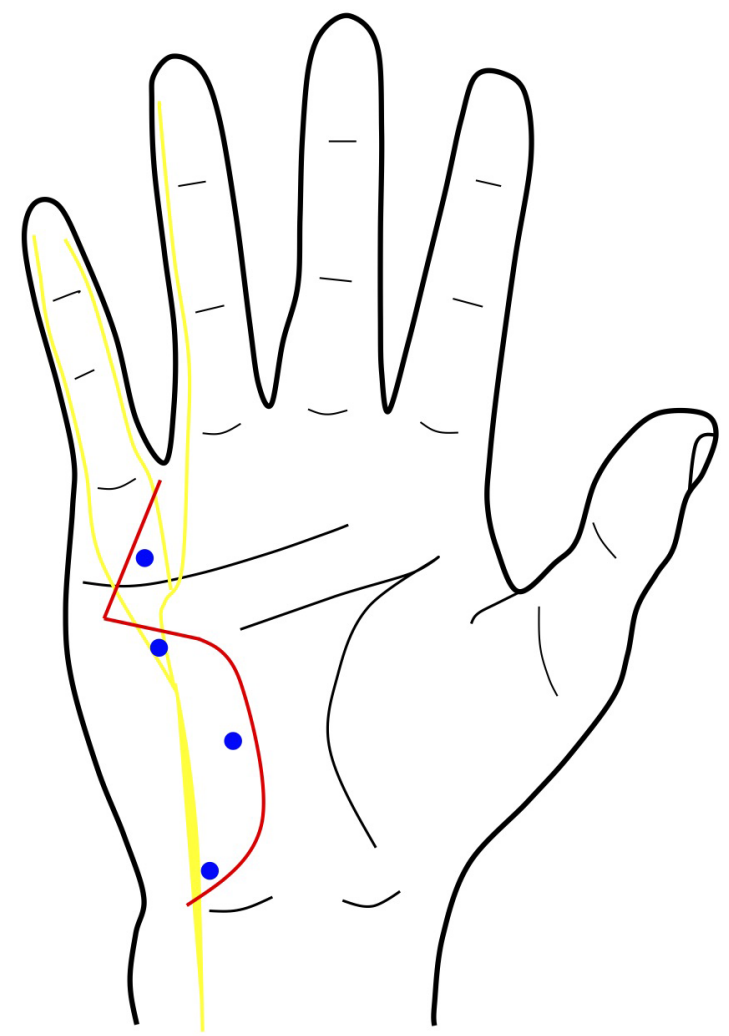

Fig. 1 The image shows the locations of the injections (blue dots) for flexor digitorum superficialis (FDS) tendon transfer. The surgeon injects 10 to $25 \mathrm{~mL}$ of local anesthetic under the incision (red line). The nerves are indicated with a yellow line.

which was identified by the three-dimensional (3D) images of the tendon ( - Fig. 2). This image was created using volume rendering of the CT scans, which were created preoperatively by the radiologists. ${ }^{8}$ The distal and proximal stumps of the ruptured FDP tendon were exposed. The proximal stump was grasped gently and pulled distally. We confirmed that the proper maximum amplitude of the proximal stump of the ruptured tendon was less than $15 \mathrm{~mm}$. Then, the ring finger FDS tendon was severed distally as much as possible and was passed behind the intervening common palmer digital artery and nerve. The proximal stump of the FDS tendon was sutured into the distal stump of the FDP tendon, using a single fine nonabsorbable suture. The tension of the transferred tendon was considerably tight when the flexion angle of the proximal interphalangeal (PIP) joint was approximately $80^{\circ}$ and the wrist was in the neutral position ( - Fig. 3). The surgeons confirmed that the patients could actively flex and fully extend their digits. After the appropriate tension was obtained, the proximal stump of the FDS tendon was weaved through the distal stump of the FDP tendon, and then perpendicular to the previous pass, for a total of two or three passes. The tendons were secured with figure-of-eight sutures at each pass and at both ends. If the tension of the transferred tendon was not appropriate, then it could be adjusted until the proper tension was achieved. Finally, the incision was closed. 


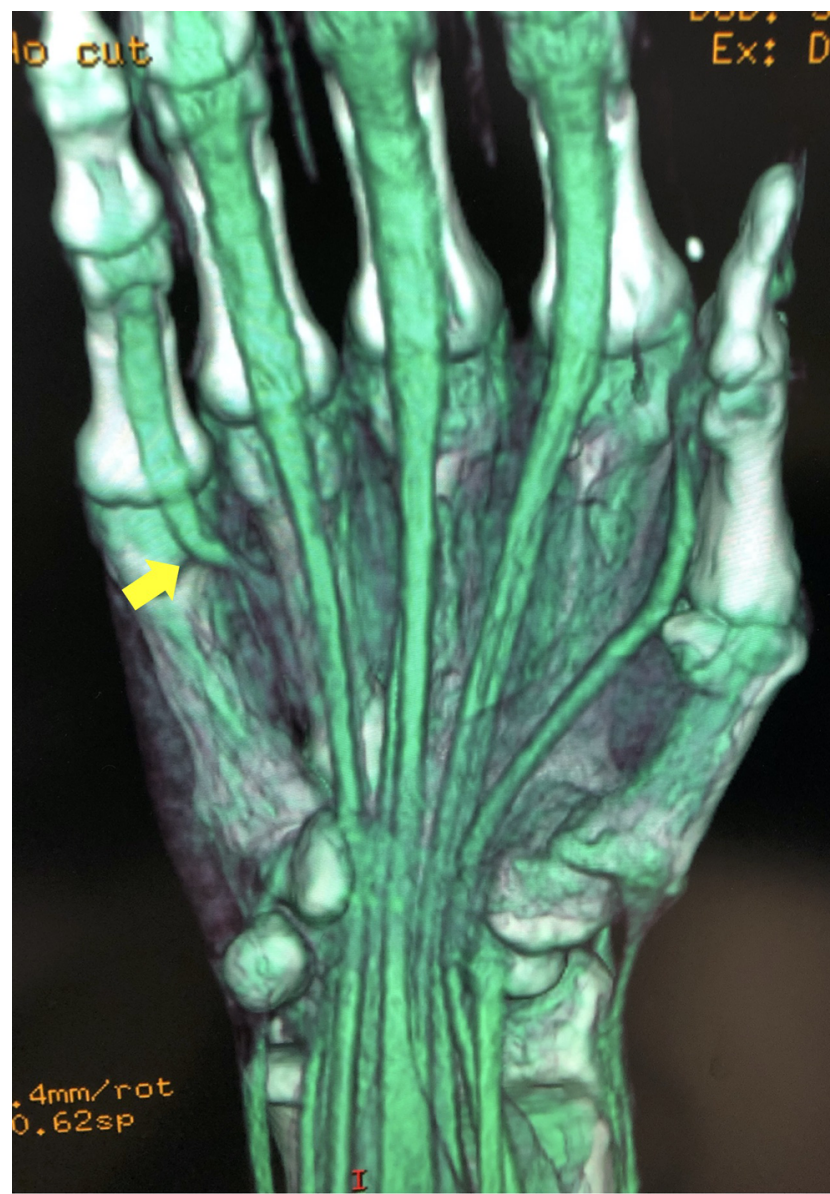

Fig. 2 A three-dimensional image of the tendon using volume rendering of the CT scans that is created preoperatively. The distal stump of the ruptured flexor digitorum profundus (FDP) tendon of the little finger is indicated with a yellow arrow. .

\section{Postoperative Treatment}

A dorsal protective splint was used to immobilize the hand, with the wrist in a neutral position, the metacarpophalangeal (MP) joints at 30 to $60^{\circ}$ of flexion, and the interphalangeal (IP) joints fully extended. All patients were hospitalized for at least 4 weeks after surgery for postoperative treatment and received instruction and supervision from the hand therapists every day. This hospitalization, which included rehabilitation, was covered by either industry injury insurance or personal health insurance. On the first postoperative day, mobilization began after the surgeon had removed the bulky dressing and applied a new thin dressing. The patient was instructed to hold the fingers in a gentle and active manner for a few seconds while flexing the IP joints passively into the palm as much as possible. Subsequently, the MP and IP joints were permitted active full extension. This active mobilization was performed three to four times per day. After the second postoperative day, unassisted active flexion exercises were allowed. In addition, controlled passive extension according to Duran's method was performed to prevent IP joint contracture. ${ }^{9}$ The dorsal protective splint was applied when the patient was not performing exercise. At night, the little finger

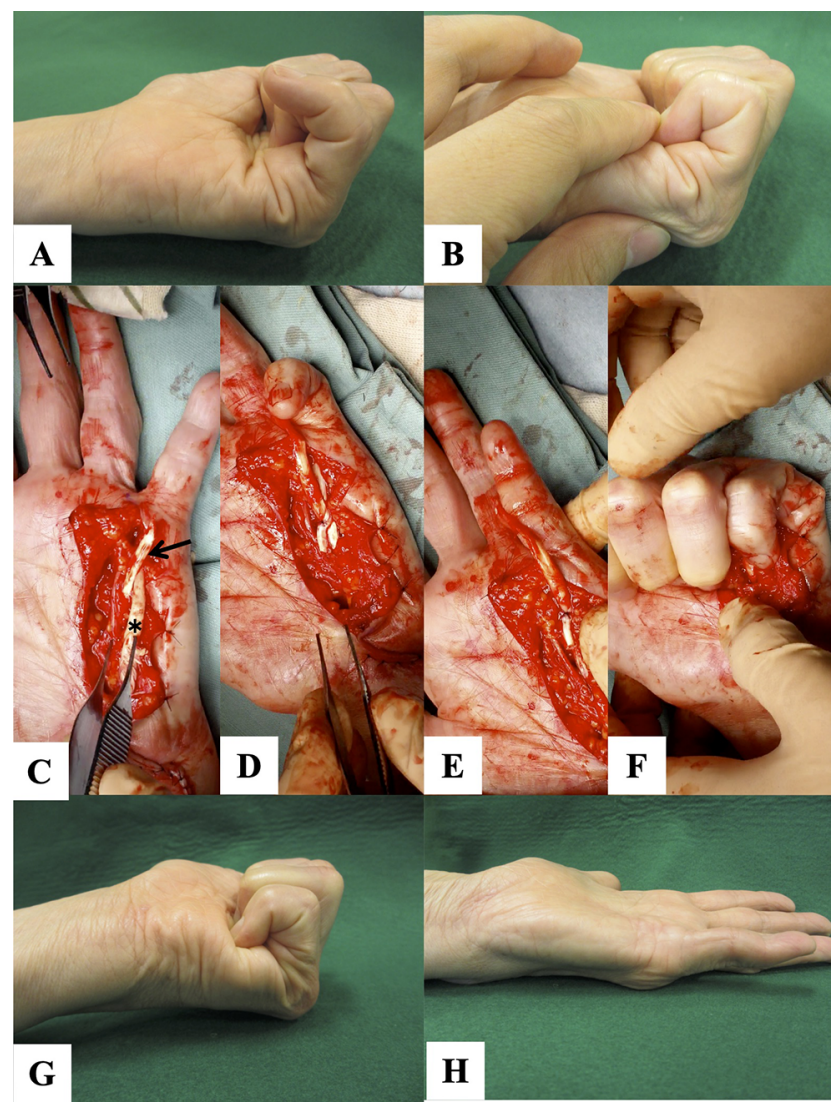

Fig. 3 Clinical images of a 78-year-old woman who sustained a zone 4 closed rupture of the flexor digitorum profundus (FDP) tendon to the little finger of her left hand. (A, B) Active and passive flexion before surgery. (C) The proximal stump of the FDP of the little finger (asterisk) and severed flexor digitorum superficialis (FDS) of the ring finger after being passed behind the intervening neurovascular bundle (arrow). (D) The transferred tendon illustrating that tension was considerably tight with an approximately $80^{\circ}$ flexion angle of the proximal interphalangeal joint (PIP) joint. (E, F) Active extension and flexion immediately after tendon transfer. $(\mathbf{G}, \mathbf{H})$ Postoperative flexion and extension 27 weeks after surgery.

was flexed, and the other digits were extended, to remove tension from the suture site of the tendon. The dorsal block splint was completely removed at 6 weeks after surgery.

\section{Outcome Measurements}

At the final evaluation, the surgeon or therapist used a goniometer to measure the active motion of the three joints of the finger, that is, metacarpophalangeal (MCP), PIP, and distal interphalangeal (DIP) joints. The clinical results were evaluated with the total active motion (TAM) of the little finger, the \%TAM, the grip strength of both arms, and a functional evaluation according to Strickland and Glogovac's criteria. ${ }^{10}$ The Quick Disability of the Arm, Shoulder, Hand-Japanese version (Quick DASH-JSSH) scores were recorded for patient-rated outcomes. ${ }^{11}$ Intraoperative findings and complications, which included grafted tendon rupture, phlegmon, or wound dehiscence after tendon grafting, were noted at the monthly visit. 


\section{Results}

\section{Number of Patients and Injured Fingers}

Thirty-four patients were diagnosed with a closed rupture of the FDP tendon of the little finger in zone 3 or 4 between January 2012 and October 2019. A total of six fingers from six patients met the criteria and were reviewed in this study.

- Table 1 shows the injury-related data. The operations were performed by three surgeons who had the following levels of expertise ${ }^{12}$ : level 4 (highly experienced specialist), $n=2$ (operated on five patients); and level 2 (less experienced specialist), $n=1$ (operated on one patient).

\section{Intraoperative Findings and Complications}

The cause of the injury was not revealed for one patient. FDP rupture of the little finger was caused by pisotriquetral osteoarthritis in three patients. An abrasive surface that resulted from nonunion of the hook of hamate caused an FDP rupture of the little finger in one patient. The lack of interdependence between the FDP tendons of the ring and little finger was found as the cause of rupture in another patient.

No wound complications or infections were noted. There were no ruptures of the transferred tendons and no fingers that required tenolysis. There were no complaints about the ring finger.

\section{Recovery and Range of Active Digital Motion}

- Table 2 shows the clinical results related to FDS tendon transfer. The mean follow-up period was 40.3 weeks (range: 27-56 weeks). The average TAM and \%TAM at the final evaluation were $201.8^{\circ}$ (range: $85-248^{\circ}$ ) and $75.4 \%$ (range: $30.4-88.1 \%$ ), respectively. At the final visit, the grip strength was, on average, $17.0 \mathrm{~kg}$ (range: $3.0-39.0 \mathrm{~kg}$ ) in the affected arm and $23.5 \mathrm{~kg}$ (range: $11.9-46.0 \mathrm{~kg}$ ) on the unaffected side. According to the Strickland and Glogovac criteria, outcomes were excellent for two, good for three, and poor for one patient. Of the six patients, only one, a 78-year-old woman, experienced a poor outcome. The patient had maintained a good range of motion during her hospital stay; however, the range of motion restriction gradually progressed after discharge, and the TAM at the final follow-up was $85^{\circ}$. The main cause of the poor TAM was the loss of extension of the PIP joint. The mean Quick DASH-JSSH score for the disability/symptom module was 14.6 (range: $0-32.5$ ).

\section{Discussion}

The current study showed satisfactory outcomes for the FDS tendon transfer, using the wide-awake approach, followed by early active mobilization for the closed rupture of the FDP tendon. The average TAM and Quick DASH-JSSH scores were relatively good, and no major complications were noted, although grip strength in some patients had not recovered sufficiently. There were no complaints regarding the donor site. We believe that using the wide-awake approach and early active mobilization may contribute to good clinical outcomes after the FDS tendon transfer.

In a previous report, adjustment of the tension of the transferred tendon was performed by observing the cascade of increasing flexion of the fingers when proceeding from the

Table 1 Data for the six patients who underwent FDS tendon transfer using the wide-awake approach

\begin{tabular}{|l|l|l|l|l|l|}
\hline Case & Age (years) & Sex & Zone of injury & FDS rupture & Mechanism of injury \\
\hline 1 & 78 & Female & 4 & None & Pisotriquetral osteoarthritis \\
\hline 2 & 74 & Female & 4 & None & Pisotriquetral osteoarthritis \\
\hline 3 & 69 & Male & 4 & None & Unknown \\
\hline 4 & 78 & Female & 4 & None & Pisotriquetral osteoarthritis \\
\hline 5 & 79 & Female & 3 & None & Variation of the FDP tendon \\
\hline 6 & 55 & Male & 4 & None & Nonunion of the hook of hamate \\
\hline Average & $72.2($ SD 8.4$)$ & & & & \\
\hline
\end{tabular}

Abbreviations: FDS, flexor digitorum superficialis; FDP, flexor digitorum profundus; SD, standard deviation.

Table 2 Clinical assessment related to FDS tendon transfer

\begin{tabular}{|l|l|l|l|l|l|l|l|}
\hline Case & $\begin{array}{l}\text { Timing of } \\
\text { surgery } \\
\text { (weeks) }\end{array}$ & $\begin{array}{l}\text { Final } \\
\text { follow-up } \\
\text { (weeks) }\end{array}$ & $\begin{array}{l}\text { Grip strength } \\
\text { of the affected } \\
\text { arm }(\mathbf{k g})\end{array}$ & $\begin{array}{l}\text { Grip strength of } \\
\text { the unaffected } \\
\text { arm }(\mathbf{k g})\end{array}$ & $\begin{array}{l}\text { TAM } \\
\text { (degrees) }\end{array}$ & $\begin{array}{l}\text { \%TAM } \\
\text { evaluation }{ }^{\text {a }}\end{array}$ \\
\hline 1 & 9 & 27 & 9.9 & 22.1 & 244 & 84.1 & Excellent \\
\hline 2 & 7 & 28 & 9.0 & 13.0 & 229 & 88.1 & Good \\
\hline 3 & 28 & 44 & 27.3 & 31.3 & 172 & 79.3 & Good \\
\hline 4 & 9 & 52 & 13.8 & 16.7 & 233 & 87.3 & Excellent \\
\hline 5 & Unknown & 35 & 3.0 & 11.9 & 85 & 30.4 & Poor \\
\hline 6 & 26 & 56 & 39.0 & 46.0 & 248 & 83.2 & Good \\
\hline
\end{tabular}

Abbreviations: FDS, flexor digitorum superficialis; TAM, total active motion.

a The Strickland and Glogovac criteria. 
index to the small finger. ${ }^{3}$ However, the appropriate tension is difficult to determine during FDS tendon transfer because the characteristics of the muscle contraction differ between the FDP and FDS. Several reports have shown that the FDS mainly works at a later phase of grip motion and is recruited during active power grip. ${ }^{13,14}$ If there is insufficient tension of the transferred FDS tendon, then during grip motion, the little finger is not flexed simultaneously with the other fingers that are mobilized by the FDP. We believe that tension of the transferred FDS tendon should be considerably increased to obtain sufficient flexion of the little finger with a slight grip motion. The considerably tight tension applied a strain to the FDS and encouraged gliding. However, there is a concern that limitation of extension remains in this situation, because surgeons cannot confirm whether full extension is possible when the patient is under a brachial plexus block or general anesthesia.

The wide-awake approach can allay this concern. Wide-awake tendon surgery has an important advantage, in that surgeons can easily determine the tension of the tendon and observe active movement of the tendon during surgery. ${ }^{7}$ Another advantage is that patients can recognize the recovery of their hand function during surgery. Moreover, the wide-awake approach could minimize the differences in outcomes that are affected by the surgeons' experiences. Thus, wide-awake surgery is particularly useful in FDS tendon transfer, in which it is difficult to determine the tension of the transferred tendon. However, considering that we used a pneumatic tourniquet for the first 20 to 30 minutes of surgery, the anesthesia technique used in this study was simple "wide-awake surgery" and not the "wide-awake local anesthesia no tourniquet" approach. ${ }^{6}$

In Japan, a prolonged hospital stay for rehabilitation after surgery is common. We ensure that patients with closed ruptures of the flexor tendon are admitted to the hospital for at least 4 weeks for postoperative treatment, because safe and controlled early active mobilization following FDS tendon transfer is also a key factor that contributes to a satisfactory outcome. The hand surgeon and therapist monitor the condition of patient's hand every day. Schneider and Wehbe reported that $25 \%$ of patients require tenolysis after FDS tendon transfer to the FDP, because active motion begins 3 to 4 weeks postoperatively. ${ }^{3}$ Koda et al suggested that the modified Kleinert method after FDP tendon transfer may contribute to a poor outcome. ${ }^{4}$ Early active mobilization can reduce the risk of developing adhesions and result in improved functional outcomes after tendon surgery; thus, this protocol has been used widely for tendon transfers such as the extensor indicis proprius to extensor pollicis longus tendon transfer. ${ }^{15,16}$ Although we employed early active mobilization after the FDS tendon transfer and achieved good outcomes, there was a patient with a poor outcome due to loss of extension of the PIP joint. This patient had maintained a good range of motion during the 4 weeks of hospitalization. Thus, for this surgical procedure, we believe that patients should undergo strict extension exercises for more than 4 weeks, which is especially important for elderly patients. Moreover, it might be better to continue extension exercises for 12 weeks, because this is the timeframe in which the scar tissue matures. ${ }^{17}$

Our study has some limitations. The major limitation was that this was a small retrospective study with no comparison group. To confirm our proposed theory, a larger case-control study is warranted. However, we believe that this might be difficult, due to the idiosyncrasies of the disease and the surgical procedure. Second, selection bias could not be excluded, because the surgical procedure was selected based on the surgeon's preference; when the proper maximum amplitude of the proximal stumps of the ruptured FDP tendon was less than $15 \mathrm{~mm}$, either tendon transfer, using the ring finger FDS tendon, or an end-to-side tendon transfer using the ring finger FDP was performed. Finally, the mean follow-up period was short (mean 40.3 weeks). A longer time of observation is necessary, considering that the tendon healing maturation phase continues for up to 1 year. ${ }^{18}$

\section{Conclusion}

We recommend the FDS tendon transfer of the ring finger, as the first-line tendon transfer within the available surgical options, when the muscle amplitude of the ruptured FDP of the little finger is insufficient. Although the FDS tendon transfer is technically challenging, the wide-awake approach is helpful to determine the tension during surgery, and postoperative early active motion exercise is also useful to obtain a satisfactory outcome.

\section{Funding}

The authors received no financial support for the research, authorship, or publication of this article.

\section{Ethical Approval}

Ethical approval for this study was obtained from the Ethics Committee of the Niigata Hand Surgery Foundation (Hospital approval number: R2-1). The study was conducted, according to the principles of the World Medical Association Declaration of Helsinki.

\section{Informed Consent}

Written informed consent was obtained from all patients before their participation in the study and for the publication of this manuscript.

\section{Conflict of Interest}

The authors declare that they have no conflicts of interest.

\section{Acknowledgments}

The authors would like to thank Dr. Takehito Takano and Dr. Masato Nakadai for comments on the manuscript.

\section{References}

1 Saito $\mathrm{H}$. Investigation of gliding amplitude of tendon as the basis of tendon surgery. J Jpn Orthop Assoc 1972;46:479-501

2 Mayer L. Repair of severed tendons. Am J Surg 1938;42:714-722

3 Schneider LH, Wehbe MA. Delayed repair of flexor profundus tendon in the palm (zone 3 ) with superficialis transfer. J Hand Surg Am 1988;13(2):227-230 
4 Koda H, Yoshizu T, Tsubokawa N. Outcome of the tendon transfer and free tendon graft for the closed rupture of the flexor tendons in Zone 3, 4. J Jpn Soc Surg Hand 2014;30:557-561

5 Yamazaki H, Kato H, Nakatsuchi Y, Murakami N, Hata Y. Closed rupture of the flexor tendons of the little finger secondary to non-union of fractures of the hook of the hamate. J Hand Surg [Br] 2006;31(3):337-341

6 Lalonde $\mathrm{DH}$. Conceptual origins, current practice, and views of wide awake hand surgery. J Hand Surg Eur Vol 2017;42(9):886-895

7 Tang JB. Wide-awake primary flexor tendon repair, tenolysis, and tendon transfer. Clin Orthop Surg 2015;7(3):275-281

8 Pelc JS, Beaulieu CF. Volume rendering of tendon-bone relationships using unenhanced CT. AJR Am J Roentgenol 2001;176(4):973-977

9 Duran RJ, Houser RG. Controlled passive motion following flexor tendon repair in zone 2 and 3. In: American Academy of Orthopaedic Surgeons Symposium on Tendon Surgery in the Hand. St. Louis: CV Mosby; 1975:105-114

10 Strickland JW, Glogovac SV. Digital function following flexor tendon repair in Zone II: A comparison of immobilization and controlled passive motion techniques. J Hand Surg Am 1980;5(6):537-543

11 Imaeda $\mathrm{T}$, Toh $\mathrm{S}$, Nakao $\mathrm{Y}$, et al. Impairment Evaluation Committee, Japanese Society for Surgery of the Hand. Validation of the Japanese Society for Surgery of the Hand version of the disability of the arm, shoulder, and hand questionnaire. J Orthop Sci 2005;10(4):353-359
12 Tang JB. Outcomes and evaluation of flexor tendon repair. Hand Clin 2013;29(2):251-259

13 Butler TJ, Kilbreath SL, Gorman RB, Gandevia SC. Selective recruitment of single motor units in human flexor digitorum superficialis muscle during flexion of individual fingers. J Physiol 2005;567(Pt 1) :301-309

14 Long C, Brown ME. Electromyographic kinesiology of the hand: muscles moving the long finger. J Bone Joint Surg Am 1964;46:1683-1706

15 Brown SH, Hentzen ER, Kwan A, Ward SR, Fridén J, Lieber RL. Mechanical strength of the side-to-side versus Pulvertaft weave tendon repair. J Hand Surg Am 2010;35(4):540-545

16 Germann G, Wagner H, Blome-Eberwein S, Karle B, Wittemann M. Early dynamic motion versus postoperative immobilization in patients with extensor indicis proprius transfer to restore thumb extension: a prospective randomized study. J Hand Surg Am 2001;26(6):1111-1115

17 Levenson SM, Geever EF, Crowley LV, Oates JF, III, Berard CW, Rosen $\mathrm{H}$. The healing of rat skin wounds. Ann Surg 1965;161:293-308

18 Moriya K, Yoshizu T, Maki Y, Tsubokawa N, Narisawa H, Endo N. Clinical outcomes of early active mobilization following flexor tendon repair using the six-strand technique: short- and long-term evaluations. J Hand Surg Eur Vol 2015;40(3):250-258 